\title{
Measuring mating preferences: the use of Manly's beta
}

\author{
Hamish G. Spencer
}

Museum of Comparative Zoology, Harvard University, Cambridge, MA 02138, U.S.A.

The use of Manly's (1985) statistic $\beta$, in mate choice experiments is discussed. Beta is a measure of the homogamic mating preference of one sex (usually females) of a strain. Beta has several advantages over previously proposed measures of deviation from random mating: (a) its sampling distribution is known and hence it can be used in establishing confidence intervals and in hypothesis testing; (b) this distribution is continuous and unimodal, even when fairly small numbers of individuals are used; (c) beta is not affected by changes in the numbers of individuals used (provided the preference remains constant); (d) it is not affected by changes in the ratios of the two strains, and (e) it is not affected by the proportions of females mated. Several properties of $\beta$ and its estimate from an experiment, $\hat{\beta}$, are derived and have been verified for a wide range of conditions using computer simulations. Their consequences for experiments attempting to quantify sexual isolation are discussed. Finally, statistics to measure the significance of $\hat{\beta}$ values are given.

\section{INTRODUCTION}

In quantifying the divergence in mating behaviour between two strains of organisms (usually Drosophila), a number of experimental designs have been used (see Parsons, 1973 and Ehrman and Parsons, 1981). The one considered in detail here is known as female choice: females of one strain are placed with males of both and the number of the two possible mating types is recorded. (There are thus two female choice experiments possible, one for each female strain). The converse experiment, in which the male and female roles are reversed, is known as male choice. The length of these experiments may differ in different designs. Many of the existing indices of sexual vigour and sexual isolation are most accurate if only about half of the individuals mate (Parsons, 1973) and so many experiments are terminated when this has occurred. Alternatively, the experiments can be run for a fixed period of time.

In addition, a variety of different indices has been used to measure deviation from panmixis. Unfortunately, the most commonly used statistics suffer from a number of undesirable statistical properties. For example, in male choice experi- ments, the Charles-Stalker index,

$$
b_{1,2}=\frac{x_{1,1} / n_{1}-x_{1,2} / n_{2}}{x_{1,1} / n_{1}+x_{1,2} / n_{2}},
$$

where $n_{j}$ is the number of strain $j$ females and $x_{i, j}$ is the number of these mated by strain $i$ males, is often used. (The symbols used are summarised in Appendix 1.) The reciprocal index $b_{2,1}$ is obtained analogously using strain 2 males. However, $b_{1,2}$ and $b_{2,1}$ are very sensitive to the proportion of females mated and hence to the duration of the experiment in which the index is measured (Levene, 1949).

Bateman's joint index, $b_{1 \text { and } 2}=\left(b_{1,2}+b_{2,1}\right) / 2$, depends not only on this proportion, but also on whether or not the proportion was the same in both the $b_{1,2}$ and $b_{2,1}$ experiments (Levene, 1949). Levene's

$$
K_{1,2}=\frac{\log \left[\left(1-x_{1,1} / n_{1}\right) /\left(1-x_{1,2} / n_{2}\right)\right]}{\log \left[\left(1-x_{1,1} / n_{1}\right)\left(1-x_{1,2} / n_{2}\right)\right]},
$$

is also sensitive to the length of time of the experiment, but to a lesser extent than $b_{1,2}$ (Levene, 1949). $K_{2,1}$ and $K_{1 \text { and } 2}$ defined in a similar manner to $b_{1,2}$ and $b_{1 \text { and } 2}$ have the same problem. 
Ideally we want an index which avoids these problems and which has some intuitive and real meaning that aids our understanding of the situation. For example, the index might enable us to predict the number of the various types of matings if we altered the ratio of the strains or increased the number of females. The statistic $\beta$ and the estimate of this obtained from an experiment, $\hat{\beta}$, discussed below appear to meet these requirements. In addition $\hat{\beta}$ has a known sampling distribution, making it amenable to various tests of significance.

\section{MANLY'S BETA: A MEASURE OF DEVIATION FROM RANDOM MATING}

Manly, Miller and Cook (1972) first introduced $\beta$ for use in the analysis of prey choice in quail. Manly (1974) extended its applicability to the situation with more than two prey items and distinguished experiments in which the prey were replaced (Type One Selection Experiments) from those in which they were not (Type Two Selection Experiments). The calculations below are derived from the latter paper. More recently Manly (1985) has applied $\beta$ to non-random mating and sexual selection and shown how $\hat{\beta}$ is related to the measures described above.

Consider a female choice experiment in which Strain 1 females are placed with $A_{1}$ Strain 1 males and $A_{2}$ Strain 2 males. Let the number of matings be $e_{1}$ and $e_{2}$ respectively. Let us assume that females mate only once and that the relative availability of males does not change over the course of the experiment. The latter will be true if there is a large excess of males over females, or that males can mate repeatedly and mating takes only a short time. In effect, the females independently sample, with replacement, the whole set of males. Such a model assumes that the females are independently and identically distributed with respect to mating behaviour. Most of the rest of this paper is restricted to the problem in which all females have identical preferences. (Where there is individual variation the situation is much more complex. The estimates of the preference parameter $(\beta)$ defined below do estimate the population means of the true values, but the various hypothesis tests are not valid.) Note that with these assumptions $e_{i}$ may be larger than $A_{1}$ for $i=1$ and 2 . Given these assumptions, it seems reasonable that the probability that a female mates homogamically will be given by

$$
\text { Prob }=\beta A_{1} /\left(\beta A_{1}+(1-\beta) A_{2}\right),
$$

where $\beta$ is a measure of the homogamic preference of Strain 1 females. If Strain 1 and Strain 2 males are equally abundant, $\beta$ is the probability that a (Strain 1) female's mating is with a Strain 1 male. Hence the probability that, given that she mates, she mates with a Strain 2 male is given by $1-\beta$. Females who do not mate at all do not affect $\beta$ (or its estimator $\hat{\beta}$ discussed below) and hence it is insensitive to any reluctance of females to mate. Beta thus ranges from 0 for completely negative assortative mating, through 0.5 for random mating, to 1 for complete positive assortative mating. Note that $\beta$ has the same value whatever the numbers or the ratios of the strains. If there is any rare male effect there will be a significant regression of $\hat{\beta}$ values on the frequency of the strains of males (Manly, 1985).

In what follows let

$$
\begin{gathered}
X=\frac{e_{1}}{A_{1}^{2}}+\frac{e_{2}}{A_{2}^{2}}, \\
Y=\frac{e_{1}}{A_{1}}+\frac{e_{2}}{A_{2}},
\end{gathered}
$$

$M=\left(e_{1}+e_{2}\right) /\left(A_{1}+A_{2}\right)$, the mean male mating fraction and $E[$ ] be the expected value.

Note that $M$ can be larger than 1. The maximum likelihood estimator of $\beta$ is given by

$$
\hat{\beta}=\frac{e_{1} / A_{1}}{e_{1} / A_{1}+e_{2} / A_{2}}=\frac{e_{1}}{A_{1} Y} \quad \text { (Manly, 1974). }
$$

If individual females of the same strain have different values, then $\hat{\beta}$ is an estimate of the mean of these values. Unfortunately, $\hat{\beta}$ is not unbiased (i.e., $E[\hat{\beta}] \neq \beta$ ), the bias' being given approximately by

$$
\operatorname{Bias}[\hat{\beta}]=\frac{\beta}{E[Y]}\left[\frac{E[X]}{E[Y]}-\frac{1}{A_{1}}\right]
$$

and its sampling variance by

$$
\operatorname{Var}[\hat{\beta}]=\beta^{2}\left[\frac{1}{E\left[e_{1}\right]}-\frac{2}{A_{1} E[Y]}+\frac{E[X]}{E[Y]^{2}}\right]
$$

(Manly, 1974). (This will not be valid if there is individual variation in $\beta$ values.) Now

$$
\begin{aligned}
& E\left[e_{1}\right]=\beta A_{1} E[Y] \\
& E\left[e_{2}\right]=(1-\beta) A_{2} E[Y]
\end{aligned}
$$

and

$$
E[Y]=\frac{E[M]\left(A_{1}+A_{2}\right)^{2}}{\beta A_{1}^{2}+A_{1} A_{2}+(1-\beta) A_{2}^{2}},
$$


(see Appendix 2 for derivation). This enables equations (1) and (2) to be reduced to

$$
\operatorname{Bias}[\hat{\beta}]=\frac{\beta(1-\beta)}{E[Y]}\left(\frac{1}{A_{2}}-\frac{1}{A_{1}}\right)
$$

and

$$
\operatorname{Var}[\hat{\beta}]=\frac{\beta(1-\beta)}{E[Y] A_{1} A_{2}}\left(A_{2}+\beta\left(A_{1}-A_{2}\right)\right) .
$$

Using the results of computer simulations, Manly (1974) found that provided $E\left[e_{1}\right]$ and $E\left[e_{2}\right]$ were larger than about 5 (Condition $\mathrm{A}$ ), his formulas (equations 1 and 2 above) were accurate and $\operatorname{Bias}[\hat{\beta}] \approx 0$. Moreover, if the interval $\beta \pm$ $3 \operatorname{Var}[\hat{\beta}]^{1 / 2}$ did not include 0 or 1 (Condition $B$ ), then $\hat{\beta}$ 's sampling distribution was approximately normal.

\section{EXPERIMENTAL ESTIMATES}

Of course, in an actual experiment one does not know $\beta$ and hence equations (6) and (7) cannot be applied directly. Manly's (1974) simulations implied that if $e_{1}$ and $e_{2}$ were both larger than about 7 (Condition $C$ ), then $\beta$ could be replaced with $\hat{\beta}, E\left[e_{1}\right]$ with $e_{1}$ and $E\left[e_{2}\right]$ with $e_{2}$ and the formulas would still be accurate. In our case these substitutions lead to

$$
\begin{aligned}
\operatorname{Bias} & =\hat{\beta}^{2}(1-\hat{\beta}) \frac{A_{1}}{e_{1}}\left(\frac{1}{A_{2}}-\frac{1}{A_{1}}\right) \\
& =\hat{\beta}^{2}(1-\hat{\beta}) \frac{\left(A_{1}-A_{2}\right)}{e_{1} A_{2}} \\
& =\hat{\beta}(1-\hat{\beta})^{2} \frac{A_{2}}{e_{2}}\left(\frac{1}{A_{2}}-\frac{1}{A_{1}}\right) \\
& =\hat{\beta}(1-\hat{\beta})^{2} \frac{\left(A_{1}-A_{2}\right)}{e_{2} A_{1}}
\end{aligned}
$$

and

$$
\begin{aligned}
\operatorname{Var} & =\hat{\beta}^{2}(1-\hat{\beta}) \frac{\left(A_{2}+\hat{\beta}\left(A_{1}-A_{2}\right)\right)}{e_{1} A_{2}} \\
& =\hat{\beta}(1-\hat{\beta})^{2} \frac{\left(A_{2}+\hat{\beta}\left(A_{1}-A_{2}\right)\right)}{e_{2} A_{1}}
\end{aligned}
$$

Manly (1974) then presented several statistical tests that hold provided Conditions $\mathrm{A}$ to $\mathrm{C}$ were satisfied. It was not clear, however, how Conditions A and B could be checked. In order to discover the accuracy of the approximations in our situation a computer simulation was performed. The program required a set of six input parameters, viz. the probability of a homogamic mating given a homogamic encounter $\left(P_{1}\right)$, the probability of a heterogamic mating given a heterogamic encounter $\left(P_{2}\right)$, the number of Strain 1 males $\left(A_{1}\right)$, the number of Strain 2 males $\left(A_{2}\right)$, the mean male mating fraction $(M)$, and the maximum value for Condition $\mathrm{C}$ to be violated. Hence $\beta=P_{1} /\left(P_{1}+P_{2}\right)$. For each set of input parameters 500 experiments were simulated and for each of these $\hat{\beta}$ was calculated and, if Condition $C$ was satisfied, its bias and variance according to equations (8) and (9). Thus the distribution of the $\hat{\beta}$ 's was obtained, together with its mean and variance, as well as the means and variances of the biases as in equation (8) and variances as in equation (9) and the number of Condition $\mathrm{C}$ violations.

Some of the results are shown in table 1. Firstly, as expected, they show that changing the values of $P_{1}$ and $P_{2}$ while keeping their ratio constant has no effect at all. Secondly, it is the total number of matings that matters and so, for example, doubling $M$ while keeping $A_{1}+A_{2}$ constant has the same effect as doubling $A_{1}+A_{2}$ and keeping $M$ constant. Of course, increasing the total number of matings decreases Bias $[\hat{\beta}](\mathrm{Col} \mathrm{10)}$ and $\operatorname{Var}[\hat{\beta}](\mathrm{Col} \mathrm{12})$, as well as the number of Condition $C$ violations (Col 16). Conditions A and B were also able to be checked because the true value of $\beta$ was known.

Thirdly, and most importantly, comparison of Columns 10 and 11 shows that equation (8) is a very good approximation of Bias $[\hat{\beta}]$. Similarly, equation (9) is an excellent estimate of $\operatorname{Var}[\hat{\beta}]$, as the congruence between Columns 9,12 and 13 shows. The variances of the distributions of means of equations (8) and (9) (not shown in the table) were in all sets less than $5 \times 10^{-5}$. The only times that the equation (9) estimate of $\operatorname{Var}[\hat{\beta}]$ was appreciably different from the "true" equation (7) value was when Condition B was violated. However this always resulted in too large an estimate, which means that most statistics used would be more conservative than expected.

The simulations also showed that the minimum $e_{1}$ and $e_{2}$ value of Condition $\mathrm{C}$ could be lowered as far as 5 (and in some cases even further) and equations (8) and (9) were still accurate. This was true even when Conditions $\mathrm{A}$ and $\mathrm{B}$ were violated although in such cases a large number of experiments also violated Condition $C$ (and were thus excluded from the analysis).

In contrast to several measures of sexual isolation (see Goux and Anxolabehere, 1980) the sampling distribution of $\hat{\beta}$ was unimodal distribution for all combinations of $M, A_{1}, A_{2}$ and $\beta$ investigated. Moreover, apart from the discontinuities 
Table 1 Parameters and results of computer simulations 1. Checks on equations (8) and (9)

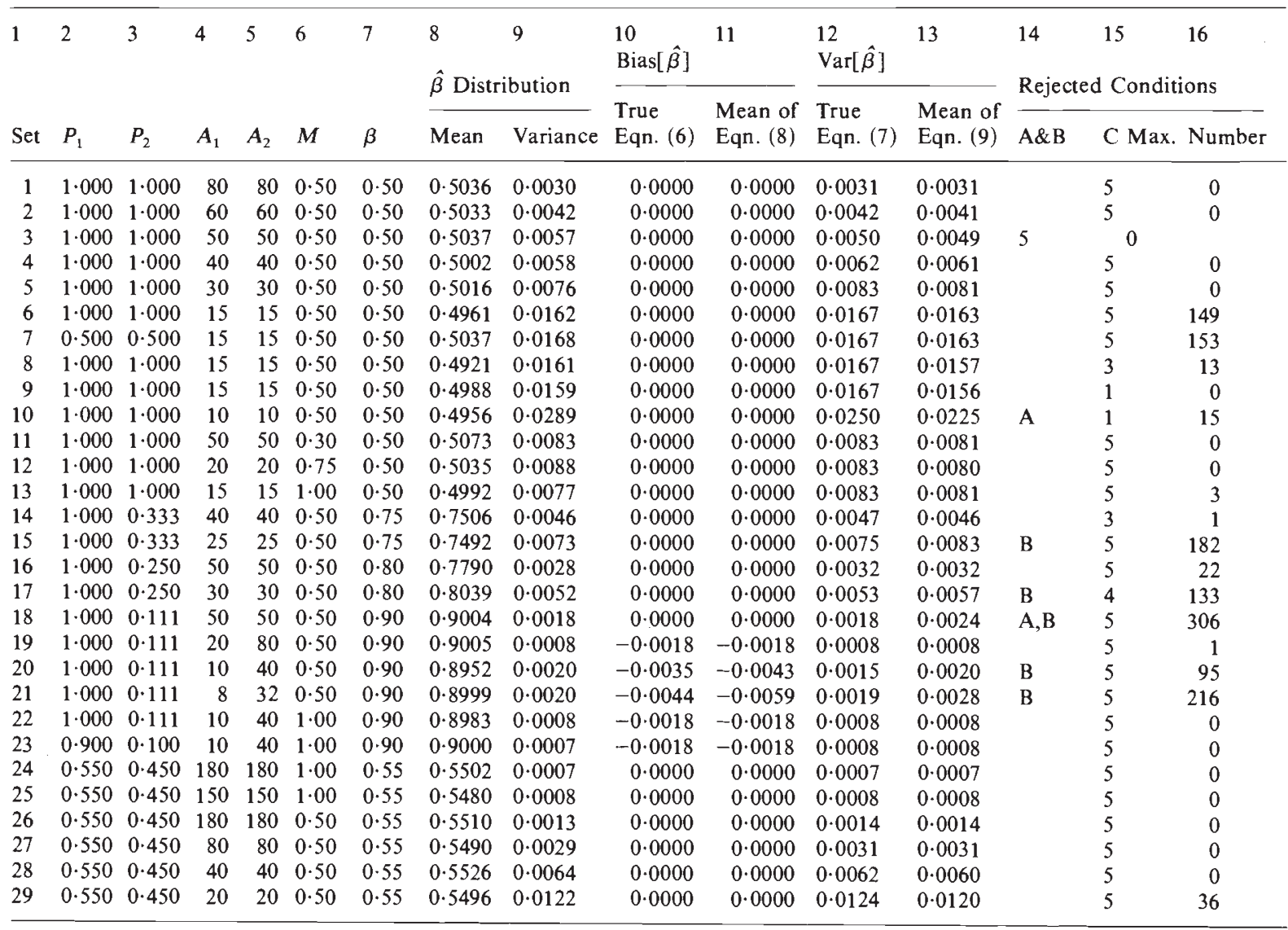

(1) A set is a simulation of 500 experiments with the corresponding parameters. (2) $P_{1}=$ Probability of a homogamic mating given a homogamic encounter. (3) $P_{2}=$ Probability of a heterogamic mating given a heterogamic encounter. (4) $A_{1}=$ Number of Strain 1 (homogamic) males. (5) $A_{2}=$ Number of Strain 2 (heterogamic) males. (6) $M=$ Mean male mating fraction= Number of matings/Total number of males. (7) True value of $\beta=P_{1} /\left(P_{1}+P_{2}\right)$. (8) Mean of set of $\hat{\beta}$ values. (9) Variance of set of $\hat{\beta}$ values.

resulting from the $e_{i}$ 's and $A_{i}$ 's being whole numbers, the distributions were continuous, the modes being as close as possible to $\beta$.

\section{CONSEQUENCES FOR EXPERIMENTAL DESIGN}

Equation (6) shows that $\hat{\beta}$ is unbiased if and only if $A_{1}=A_{2}, \beta=0$ or $\beta=1$. The last two cases are unlikely to occur in practice. However, if equal numbers of Strain 1 and 2 males are used, i.e. if $A_{1}=A_{2}$, then not only is $\hat{\beta}$ unbiased, but $E[Y]=$ $2 E[M]$ and hence

$$
\operatorname{Var}[\hat{\beta}]=\frac{\beta(1-\beta)}{2 E[M] A_{1}}=\frac{\beta(1-\beta)}{E\left[e_{1}\right]+E\left[e_{2}\right]} .
$$

However, if $\beta$ is not equal to 0.5 then, with a fixed total number of males, using equal numbers of males of each strain does not result in the minimum $\operatorname{Var}[\hat{\beta}]$. It is better to use more males of the strain less likely to mate, usually Strain 2 . Although this results in a biased estimate, my simulations show that the bias is very small if Condition $\mathrm{C}$ is satisfied (see table 1, Sets 18-23). Furthermore, using an unequal number of males considerably reduces the number of Condition $\mathrm{A}, \mathrm{B}$, and $\mathrm{C}$ violations and hence the significance of the estimate can be more easily tested.

The expression for $E[Y]$, equation (5), shows that, for a fixed total number of males, $E[Y]$ is directly proportional to $M$, the mean male mating fraction, which in turn will increase as the length of the experiment increases. $M$ will also increase as the number of females is increased. However, using many more females than males may violate the assumption that males are able to mate repeatedly and may thus change the model into one of sampling without replacement. Inspection of equations $(6)$ and $(7)$ reveals that both Bias $[\hat{\beta}]$ 
Table 2 Parameters and results of computer simulations II. Checks on hypothesis tests

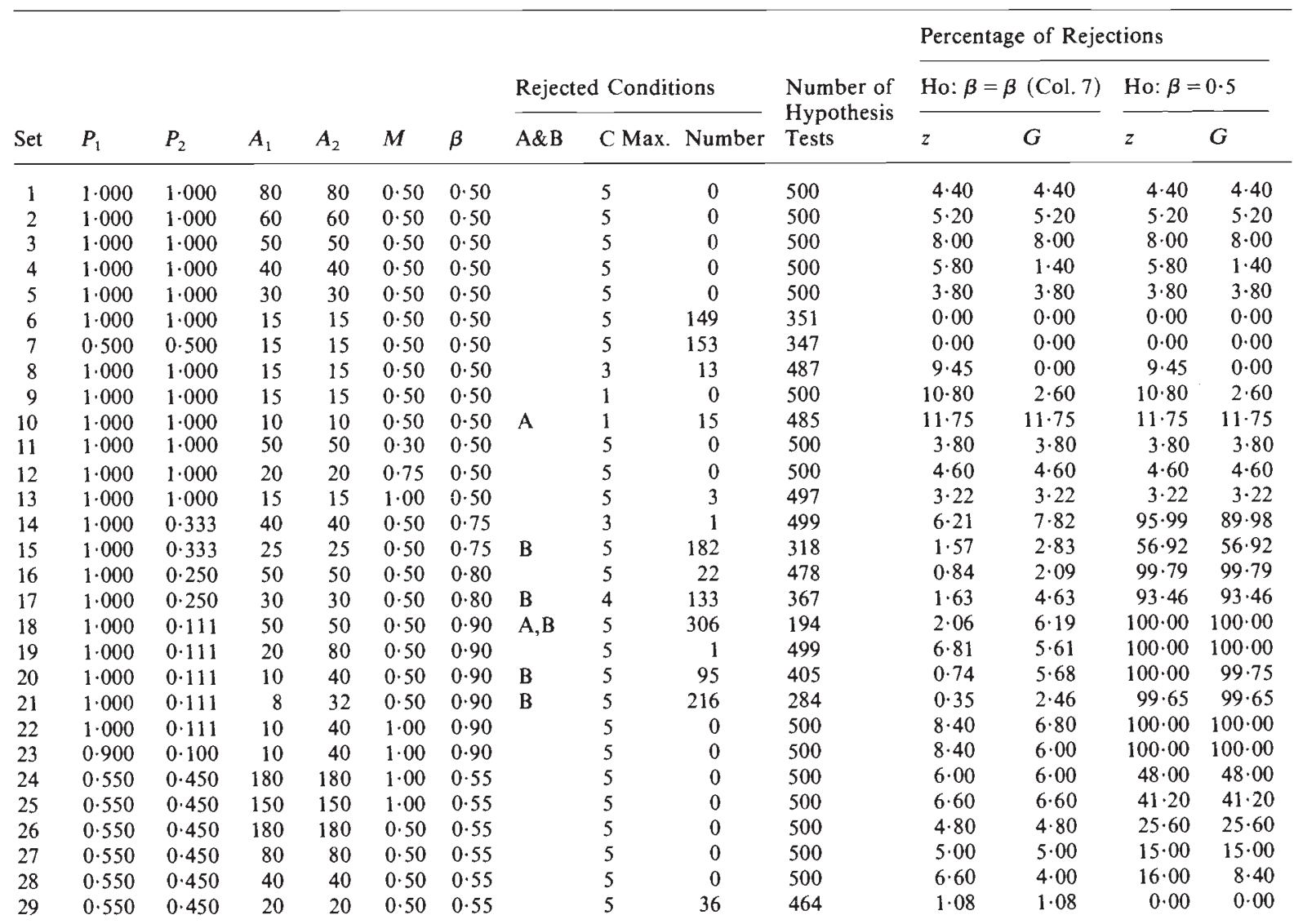

and $\operatorname{Var}[\hat{\beta}]$ are inversely proportional to $E[Y]$ and hence $M$. This means that with longer experiments more accurate results are obtained. This is an interesting contrast to the $b$ 's and $K$ 's above which are most accurate if $M$ is approximately 0.5 (Parsons, 1973).

Finally, an increase in the total number of males also decreases $\operatorname{Var}[\hat{\beta}]$, as might be expected.

Hence in choice experiments better estimates are obtained by

(a) biasing the ratio of males in favour of those less likely to mate;

(b) letting the experiment run for as long as possible, even to the extent of allowing all the females to mate, and

(c) using as many individuals of both sexes as possible.

\section{HYPOTHESIS TESTS}

To test the null hypothesis that $\beta$ is equal to a given value, $\beta^{*}$, e.g. $\beta^{*}=0.5$ for random mating, use either $(\mathrm{a}) z=\left(\hat{\beta}-\beta^{*}\right) / \operatorname{Var}^{1 / 2}$ which is standard normal (Manly, 1974), or (b) a goodness of fit $G$ test (Sokal and Rohlf, 1981), $G=$ $\sum_{i=1}^{2} e_{i} \ln \left(e_{i} / E\left[e_{i}\right]\right)$ which is Chi-square with 1 degree of freedom. The value for Var is given by any one of the forms of equation (9) and $E\left[e_{i}\right]$ is found by substituting $\hat{\beta}$ for $\beta$ and $M$ for $E[M]$ in equations (3), (4) and (5). An $\alpha$-level confidence interval of the form $\hat{\beta} \pm z_{\alpha} \operatorname{Var}^{1 / 2}$, where $z_{\alpha}$ is the appropriate $\alpha$-level standard normal value, can also be constructed.

The computer program also tested the accuracy of both these tests under two null hypotheses and the results are shown in table 2 . The level of significance of the tests was checked by testing the null hypothesis that $\beta$ was equal to the true value as given by $\beta=P_{1} /\left(P_{1}+P_{2}\right)$ and shown in Column 7 of the table. The nominal level of significance was 5 per cent and provided the Condition $\mathrm{C}$ parameter was at least 5 , most of the tests were somewhat conservative. Of course, because the sampling distribution of $\hat{\beta}$ is discrete, the true level of significance is very rarely exactly 5 per cent. Neither $z$ nor $G$ is noticeably better; indeed the 
differences between them are again due to $\hat{\beta}$ 's discrete sampling distribution. It should be noted that when Condition B was violated there was often a correspondingly high number of Condition $\mathrm{C}$ violations. However, if Condition $\mathrm{C}$ was satisfied there was a loss of power and the true level of significance declined.

The second null hypothesis was designed to test the power of the tests by testing the null hypothesis that mating was at random, i.e. $\beta=0 \cdot 5$. Again there is little to choose between $z$ and $G$, although perhaps $z$ is a little better. Not surprisingly, when $\beta$ was close to $0 \cdot 5$, a rather large sample size was necessary to avoid a Type II error.

Hence it is recommmended that these tests be used only when both $e_{1}$ and $e_{2}$ exceed 5 (i.e., Condition $C$ parameter at least 5). Secondly, if the interval $\hat{\beta} \pm 3 \operatorname{Var}[\hat{\beta}]^{1 / 2}$ includes either 0 or 1 any conclusions drawn from a failure to reject the null hypothesis under consideration should be regarded with extreme caution.

If $S$ independent experiments are conducted giving estimates $\hat{\beta}_{1}, \hat{\beta}_{2}, \ldots, \hat{\beta} s$ then we can also test the null hypothesis that they are all estimates of the same $\beta$, viz., $\beta^{*}\left(H o: \beta_{j}=\beta^{*} j=1,2, \ldots, S\right)$ e.g., $\beta^{*}=\sum_{j=1}^{s} \hat{\beta}_{j} / S$. Use $X^{2}=\sum_{j=1}^{s}\left(\hat{\beta}_{j}-\beta^{*}\right)^{2} / \operatorname{Var}_{j}$ which is Chi-square with $S-1$ degrees of freedom (Manly, 1974).

Acknowledgements I am indebted to Brian McArdle for first suggesting that beta might be useful in mate choice experiments. Rob Dorit, Dick Lewontin, Brian McArdle, Peter Taylor and an anonymous reviewer provided helpful criticism. Bryan Manly kindly supplied me with an advance copy of the relevant section of his book. The computer simulations were supported by DOE Grant No. DE-AC02-76EV02472 to R. C. Lewontin.

\section{REFERENCES}

EHRMAN, L. AND PARSONS, P. A. 1981. Behaviour Genetics and Evolution. McGraw-Hill, New York.

GOUX, J. M. AND ANXOLABEHERE, D. 1980. The measurement of sexual isolation and selection: a critique. Heredity, 45 , 255-262.

LEVENE, H. 1949. A new measure of sexual isolation. Evolution, $3,315-321$.

MANLY, B. J. F. 1974. A model for certain types of selection experiments. Biometrics, 30, 281-294.

MANLY, B. J. F. 1985. The Statistics of Natural Selection. Chapman and Hall, London.

MANLY, B. J. F., MILLER, P. AND COOK, L. M. 1972. Analysis of a selective predation experiment. American Naturalist, $106,719-736$.
PARSONS, P. A. 1973. Behavioural and Ecological Genetics: a study in Drosophila. Clarendon Press, Oxford.

SOKAL, R. R. AND ROHLF, F. J. 1981. Biometry. W. H. Freeman, San Francisco.

\section{APPENDIX 1}

$x_{i, j} \quad$ Number of strain $j$ females mated by strain $i$ males.

$n_{j} \quad$ Number of strain $j$ females.

$N$ Total number of mated females $\left(\sum_{i, j} x_{i, j}\right)$.

$e_{i} \quad$ Number of (usually strain 1) females mated by strain $i$ males.

$A_{i} \quad$ Number of strian $i$ males.

$Y=\frac{e_{1}}{A_{1}}+\frac{e_{2}}{A_{2}}$.

$M=\frac{e_{1}+e_{2}}{A_{1}+A_{2}}$, the mean male mating fraction.

$\hat{\beta}=\frac{e_{1}}{A_{1} Y}$, estimator of $\beta$.

Condition A: $E\left[e_{i}\right]>5$ for $i=1,2$.

Condition $\mathrm{B}: 0<\beta \pm 3 \operatorname{Var}[\hat{\beta}]^{1 / 2}<1$.

Condition $\mathrm{C}$ : $e_{i}>7$, although this value could be lowered to 5 .

\section{APPENDIX 2}

Since $M=\left(e_{1}+e_{2}\right) /\left(A_{1}+A_{2}\right)$, we have

$$
e_{1}=M\left(A_{1}+A_{2}\right)-e_{2}
$$

and so,

$$
\begin{aligned}
E\left[e_{1}\right] & =E[M]\left(A_{1}+A_{2}\right)-E\left[e_{2}\right] \\
& =E[M]\left(A_{1}+A_{2}\right)-A_{2}(1-\beta) E[Y] .
\end{aligned}
$$

Similarly, $\quad E\left[e_{2}\right]=E[M]\left(A_{1}+A_{2}\right)-A_{1} \beta E[Y]$

Now,

$$
\begin{aligned}
E[Y]= & \frac{E\left[e_{1}\right]}{A_{1}}+\frac{E\left[e_{2}\right]}{A_{2}} \\
= & E[M]\left(\frac{A_{1}+A_{2}}{A_{1}}+\frac{A_{1}+A_{2}}{A_{2}}\right)-E[Y] \\
& \times\left((1-\beta) \frac{A_{2}}{A_{1}}+\beta \frac{A_{1}}{A_{2}}\right) \\
= & \frac{E[M]\left(A_{1}+A_{2}\right)^{2}}{\beta A_{1}^{2}+A_{1} A_{2}+(1-\beta) A_{2}^{2}} .
\end{aligned}
$$

